\title{
Erratum: First Dark Matter Constraints from a SuperCDMS Single-Charge Sensitive Detector [Phys. Rev. Lett. 121, 051301 (2018)]
}

R. Agnese, T. Aralis, T. Aramaki, I. J. Arnquist, E. Azadbakht, W. Baker, S. Banik, D. Barker, D. A. Bauer, T. Binder, M. A. Bowles, P. L. Brink, R. Bunker, B. Cabrera, R. Calkins, C. Cartaro, D. G. Cerdeño, Y.-Y. Chang, J. Cooley, B. Cornell, P. Cushman, P. C. F. Di Stefano, T. Doughty, E. Fascione, E. Figueroa-Feliciano, C. Fink, M. Fritts, G. Gerbier, R. Germond, M. Ghaith, S. R. Golwala, H. R. Harris, Z. Hong, E. W. Hoppe, L. Hsu, M. E. Huber, V. Iyer, D. Jardin, C. Jena, M. H. Kelsey, A. Kennedy, A. Kubik, N. A. Kurinsky, R. E. Lawrence, J. V. Leyva, B. Loer, E. Lopez Asamar, P. Lukens, D. MacDonell, R. Mahapatra, V. Mandic, N. Mast, E. H. Miller, N. Mirabolfathi, B. Mohanty,

J. D. Morales Mendoza, J. Nelson, J. L. Orrell, S. M. Oser, W. A. Page, R. Partridge, M. Pepin, A. Phipps, F. Ponce, S. Poudel, M. Pyle, H. Qiu, W. Rau, A. Reisetter, T. Reynolds, A. Roberts, A. E. Robinson, H. E. Rogers, R. K. Romani, T. Saab, B. Sadoulet, J. Sander, A. Scarff, R. W. Schnee, S. Scorza, K. Senapati, B. Serfass, J. So, D. Speller, C. Stanford, M. Stein, J. Street, H. A. Tanaka, D. Toback, R. Underwood, A. N. Villano, B. von Krosigk, S. L. Watkins, J. S. Wilson, M. J. Wilson, J. Winchell, D. H. Wright, S. Yellin, B. A. Young, X. Zhang, and X. Zhao

(Received 18 January 2019; published 11 February 2019)

DOI: 10.1103/PhysRevLett.122.069901
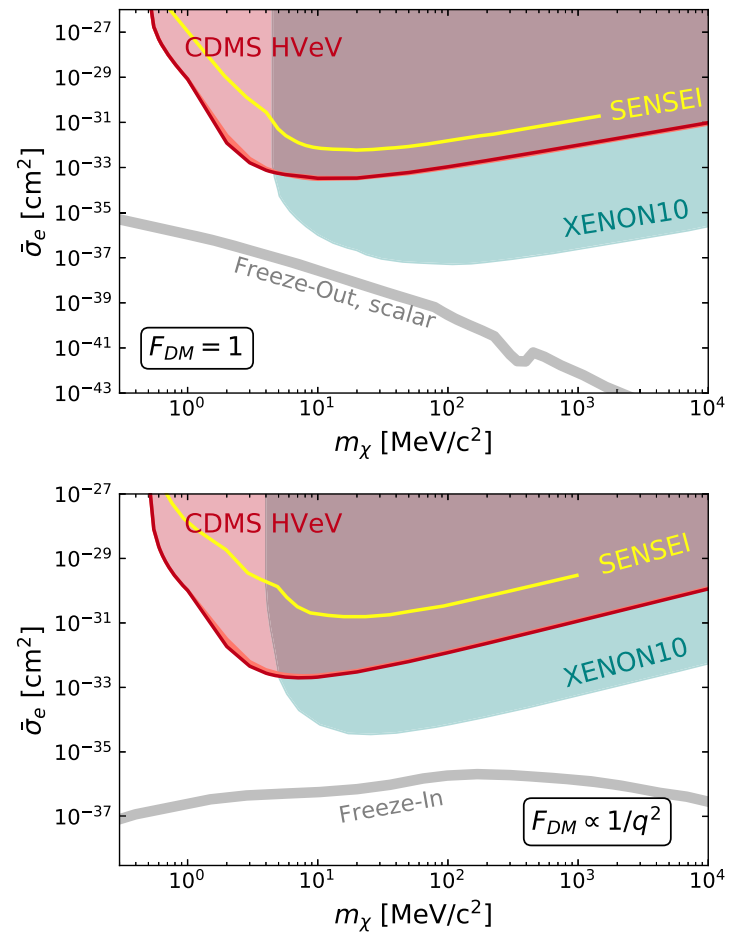

FIG. 1. Corrected limit on DM particle interacting with electrons via a heavy dark photon (top, $F_{\text {DM }}=1$ ) or an ultralight dark photon (bottom, $F_{\mathrm{DM}} \propto 1 / q^{2}$ ) compared to the XENON10 and SENSEI results [2,3]. The red line is the limit curve with a Fano factor of 0.155 in the ionization model. The salmon colored region indicates the systematic uncertainties due to varying the Fano factor between the lowest mathematically possible value and 1. For signal models as well as additional astrophysical constraints, see Ref. [4]. All limits as shown here assume a local DM density of $0.3 \mathrm{GeV} / \mathrm{cm}^{3}$.

Published by the American Physical Society under the terms of the Creative Commons Attribution 4.0 International license. Further distribution of this work must maintain attribution to the author(s) and the published articles title, journal citation, and DOI. 




FIG. 2. Total rates $R\left(m_{\chi}\right)$ of DM-electron scattering in silicon for two DM form factors, $F_{\mathrm{DM}}$, corresponding to different DM models. The blue dashed (green solid) line assumes a heavy (ultralight) dark photon mediator. The rates are the yearly average for a local DM density of $0.3 \mathrm{GeV} / \mathrm{cm}^{3}$ and are calculated with QEdark [1].

In our Letter describing the search for dark matter (DM) using a cryogenic Si chip sensitive to single electron-hole pairs, the differential scattering rate of DM particles with electrons for a given DM particle mass $m_{\chi}, d N / d E\left(m_{\chi}\right)$, was computed using the output of the publicly available QEdark notebook [1]. From this notebook we obtained an array in $0.2 \mathrm{eV}$ bins of $\Delta N_{i}$ - the expected number of events in bin $i$-for a fixed dark matter density, data acquisition time, detector mass, and cross section. In our linear approximation $\Delta N_{i}$ is related to $d N / d E$ as $\Delta N_{i} \approx d N / d E \times \Delta E_{i}=d N / d E \times 0.2$, with the recoil energy $E$ taken to have units of $\mathrm{eV}$; so $d N / d E \approx 5 \Delta N_{i}$. Because of a miscommunication, this factor of 5 binning correction was applied twice, yielding a $d N / d E$ that was 5 times higher than it should have been and an upper limit cross section that was 5 times too strong.

An updated version of Fig. 4 middle and bottom of the Letter is provided in this Erratum as Fig. 1. These new figures also include the limits observed by the SENSEI Collaboration which were published simultaneously with our original Letter in Ref. [2]. For ease of future comparison, Fig. 2 has been added to this Erratum showing the total DM-electron scattering rate in silicon at $\bar{\sigma}_{e}=10^{-37} \mathrm{~cm}^{2}$ for the probed DM models. This rate was calculated with the QEdark notebook downloaded on October 28, 2018 and forms the basis of the results shown in Fig. 1. The notebook has not changed since the publication of our Letter.

We thank Rouven Essig for useful discussions.

[1] Direct detection of sub-gev dark matter (2018), http://ddldm.physics.sunysb.edu/ddlDM/.

[2] M. Crisler, R. Essig, J. Estrada, G. Fernandez, J. Tiffenberg, M. S. Haro, T. Volansky, and T.-T. Yu (SENSEI Collaboration), SENSEI: First Direct-Detection Constraints on sub-GeV Dark Matter from a Surface Run, Phys. Rev. Lett. 121, 061803 (2018).

[3] R. Essig, A. Manalaysay, J. Mardon, P. Sorensen, and T. Volansky, First Direct Detection Limits on Sub-GeV Dark Matter from XENON10, Phys. Rev. Lett. 109, 021301 (2012).

[4] M. Battaglieri et al., US Cosmic Visions: New Ideas in Dark Matter 2017: Community Report, arXiv:1707.04591. 